\title{
基于生态系统服务视角的“土地分离与 共享框架”解析
}

\author{
冯喆 ${ }^{1,2}$, 许学工 $2^{2 *}$, 周建 ${ }^{3,4}$, 高阳 ${ }^{3,4}$ \\ (1. 中国地质大学(北京)土地科学技术学院,北京 100083 ; \\ 2. 北京大学城市与环境学院,地表过程与模拟教育部重点实验室,北京 $100871 ;$ \\ 3. 中国农业大学资源与环境学院土地资源管理系, 北京 100193 ; \\ 4. 国土资源部农用地质量与监控重点实验室, 北京 100193)
}

\begin{abstract}
摘 要: “土地分离和共享框架”是协调物质生产和生态保护、促进土地可持续利用的重要理论。本文梳理了“土地 分离与共享框架” 和生态系统服务间关联, 剖析生态系统服务应用于该框架的理论基础,结合美国典型土地共享案 例, 分析了土地利用策略选择对粮食生产、生物多样性、直接和间接生态系统服务的影响, 并探讨了该框架对中国 生态系统工程的启示。结果表明:随着人类对土地功能认知的不断深人, “土地分离与共享框架” 的拓展表现为从 单一的粮食供给服务需求演进到粮食供给服务和其他生态系统服务的综合需求。当粮食供给服务与其他服务处 于权衡状态时, 在生态脆弱、恢复力差的地区, 不同生态系统服务间多处于凸权衡关系,一般适宜采用分离策略; 在 生态系统稳定、恢复力强的地区, 不同生态系统服务多处于凹权衡关系,一般适宜采用共享策略。结合美国典型土 地共享案例发现,其土地利用实践中选择土地功能共享策略是比较适宜的;生态系统服务作为政策评估工具,可在 策略选择和评估实施效果中发挥一定作用。研究结果可为可持续土地利用提供理论与方法支撑,并为中国制定土 地利用策略提供参考。
\end{abstract}

关 键 词: 土地分离与共享; 生态系统服务;生物多样性;粮食生产;权衡

\section{1 引言}

土地利用是人类活动对生态系统加以影响的 直接表现, 反映了人类社会与植被、土壤、水资源等 自然生态系统之间交互作用的最紧密关系。科学 选择土地利用方式是协调物质生产和生态保护, 促 进土地可持续发展的重要议题。英国学者 Green 等 在 2005 年提出了 “土地分离与共享框架” (land sparing versus sharing framework), 从粮食供给和生物多 样性角度出发, 探讨有限空间内的土地利用方式选 择问题(Green et al, 2005), 该框架视角新颖、实践性 强,一经提出便引起了广泛关注。
随着研究的不断深人,仅从粮食供给和生物多 样性的角度讨论土地利用策略, 已经不能涵盖人类 对土地利用的全部需求。首先, 除粮食外, 土地还 可以供给能源纤维或作物, 咖啡、可可脂等经济作 物, 以及豆类、牧草等饲料; 其次, 生物多样性仅是 区域生态系统状况的一种重要的表征方式,并不能 完全表现生态系统的全部要素(von Wehrden et al, 2014); 第三, 在该框架中, 土地仅作为生产活动的 承载,其社会文化属性被忽视(Fischer et al, 2008)。

生态系统服务研究的发展为全面度量土地功 能、测度生态系统状况、改进和拓展“土地分离与共 享框架”提供了理论依据。生态系统服务产生于生

收稿日期:2015-10; 修订日期:2016-07。

基金项目: 国家自然科学基金项目 (41271102,41501087) [Foundation: National Natural Science Foundation of China, No.41271102, No.41501087]

作者简介: 冯吉(1984-), 男, 北京人, 博士, 讲师, 研究方向为土地利用和生态系统服务, E-mail: fengzhe@pku.edu.cn。 通讯作者:许学工(1950-),博士,教授,主要研究方向为土地利用和自然保护,E-mail: xxg@urban.pku.edu.cn。 
态环境与人类相互作用过程中, 是生态系统对人类 福祉的贡献, 形成了人类生存和发展的基础 (Costanza et al, 1997)。从本质上看, “土地分离与共 享框架”的核心是衡量不同强度的土地利用下生态 系统的综合状况, 是生态系统供给服务与调节、支 持服务间权衡关系的体现; 从实践上看, 是人类对 土地不同生态功能间的权衡取舍问题。越来越多 的学者呼吁从生态系统服务的视角解释、完善和拓 展“土地分离与共享框架”(Lerouge et al, 2016)。

本文梳理了 “土地分离与共享框架” 和生态系 统服务间关联, 剖析生态系统服务应用于该框架的 理论基础。在中国的土地利用实践中, 大多采用 “土地分离” 的策略, 如划定自然保护区等, 对 “土地 共享”的策略应用不多。因此, 本文特选取了美国 典型土地共享案例, 从生态系统服务视角着重探讨 不同土地利用方式选择对粮食供给、生物多样性、 其他直接或间接生态系统服务的影响, 旨在为土地 利用优化与生态系统服务关系研究提供借鉴。

\section{2 “土地分离与共享框架”概述}

“土地分离与共享” 又称为 “土地分离与野生动 植物友好型耕作(wildlife-friendly farm)”, 由英国学 者 Green 等(2005)在 2005 年提出。经过十几年的发 展, 研究内容涉及功能景观(Huang et al, 2015)、尺度 效应(Fischer et al, 2014)、产权制度(Boisvert, 2015)、 市场激励机制(Merckx et al, 2014)、土地利用沿革 (von Wehrden et al, 2014)等多方面, 并在非洲东部 (Habel et al, 2013)、印度(Phalan, Onial et al, 2011)、 哥斯达黎加(Fischer et al, 2008)、西班牙(Benayas et $\mathrm{al}, 2012$ )等地均有实践。

土地分离(land sparing)是指划分出部分土地不 予耕作而专用于生态系统保育和自然保护, 并采用 高效集约的利用方式提高剩余土地单产, 实现粮食 生产和自然保护的双赢(Green et al, 2005)。自然保 护区、生态控制红线、草库伦、轮耕制度等都是土地 分离策略的典型代表。土地共享(land sharing)与土 地 “多功能利用” 的概念相近, 是指对土地进行综合 利用, 不划分禁耕区, 采用较低效率的土地生产方 式,在农业生产的同时实现生物多样性保护(Green et al, 2005)。土地分离策略认为, 环境敏感、种群稀 少的脆弱物种易受人类活动影响, 因此必须开辟专 门的区域对其进行保护; 土地共享策略则认为, 分
离策略为保证粮食产量,在剩余土地上进行高集约 的农业生产, 会对这些土地上的生物多样性以及一 些非市场化的环境和社会价值造成消极影响(Law et al, 2015)。但土地共享策略所主张的低生产率耕 作方式, 因为会造成粮食减产, 常常遭到农场主、投 资人等利益相关者的反对 (Cunningham et al, 2013)。由于不能提高土地生产率, 在目前人口增 长的压力之下, 为满足日益增长的粮食需求, 可能 需要开垦更多的荒地或侵占域外森林、草地等进行 粮食生产(Lusiana et al, 2012), 也会造成环境损失。 如何更为全面地反映两种策略的优劣,判定“分离” 或“共享”策略选择标准, 已成为学界研究的重要议 题(Lerouge et al, 2016)。

大量研究试图揭示土地分离、土地共享或者混 合策略选择背后的规律, 如使用样地观测(Edwards et al, 2010; Yoshii et al, 2015; Montoya-Molina et al, 2016)、模型构建(Lusiana et al, 2012; Newbold et al, 2014)或情景模拟(Egan et al, 2012; Cardador et al, 2015)等方法测度和比较不同策略的实施成果。目 前, 土地利用策略的选择还没有统一的标准。如 Ramankutty等(2012)认为热带地区适用土地分离策 略,温带地区适用土地共享策略, 但巴西作为热带 国家,土地管理当局采用了“分离”与 “共享”结合的 策略(Scariot, 2013)。

\section{3 基于生态系统服务的“土地分离与 共享”策略选择}

\section{1 “土地分离与共享框架”的理论基础}

不同土地利用策略的效应直接表现为生态系 统功能与服务价值的变化。“土地分离与共享框架” 的核心是衡量不同土地利用强度下生态系统的综 合状况, 该框架在作用机制、策略本质、研究内容及 方法方面均与生态系统服务紧密相连。

(1) 从作用机制上看, 土地是生态系统服务的 承载。土地利用变化不仅改变生态系统的外观,而 且影响土壤水分、养分和土壤侵蚀等物质循环和能 量流动的生态过程(傅伯杰, 2013), 进而直接或间接 地引起淡水供给、有机质它存、碳咜存、土壤保持、 粮食供给等生态系统服务的改变。如从林地或草 地转变为耕地的过程,会削弱当地土壤水分涵养与 水质调节功能(傅伯杰等, 2014)。某类或某些生态 系统服务的变化也会影响土地利用,如土壤保持功 
能下降后形成石漠化或沙化区域, 导致土地利用转 变; 淡水供给服务的匮乏导致城市的衰落; 传粉、害 虫防治和养分循环等生态系统服务会影响耕地分 布等(左太安, 2014; 戴漂漂等, 2015; 刘卫, 2015)。 土地利用与生态系统服务间相互作用、相互影响的 关系, 是从生态系统服务视角审视两种土地利用策 略的基础。

(2) 从策略本质上看, 土地利用是人类活动与 自然环境综合作用的结果, 土地分离与共享策略选 择是供给和支持两种生态系统服务之间的权衡。 图 1 是对传统 “土地分离与共享框架”的拓展: 粮食 生产可进一步拓展至牧草生产服务、纤维生产服务 等; 以生物多样性为代表的环境维持属于支持服 务, 但同时包含休闲娱乐和精神归属等因素, 属文 化服务范畴。从生态系统服务角度审视“土地分离 与共享框架”, 最终目的是通过选择适宜的土地利 用方式实现多种服务的共赢。

(3) 从研究内容上看, 粮食生产和生物多样性 是土地分离与共享框架的主要内容, 也是生态系统 服务研究范畴。依据千年生态系统评估的分类体 系 (Millennium Ecosystem Assessment, 2005), 粮食 生产属于供给服务, 并与传粉、害虫防治、养分循环 等调节服务相关; 生物多样性可划归为支持服务, 也与土壤保持、生境维持等服务间接相关。

(4) 从研究方法上看, 在土地分离与共享策略 的效用评估中,粮食生产常采用粮食总产量及其市 场价值估计; 生物多样性常用群落内多样性 $(\alpha$ 多样 性)评价, 也有少量使用群落间多样性 ( $\beta$ 多样性)进 行评价。生态系统服务评估同样包括对粮食供给 服务和生物多样性的度量, 此外还有多种模型方法 对土壤保持、碳咜存等服务进行度量, 从生态系统

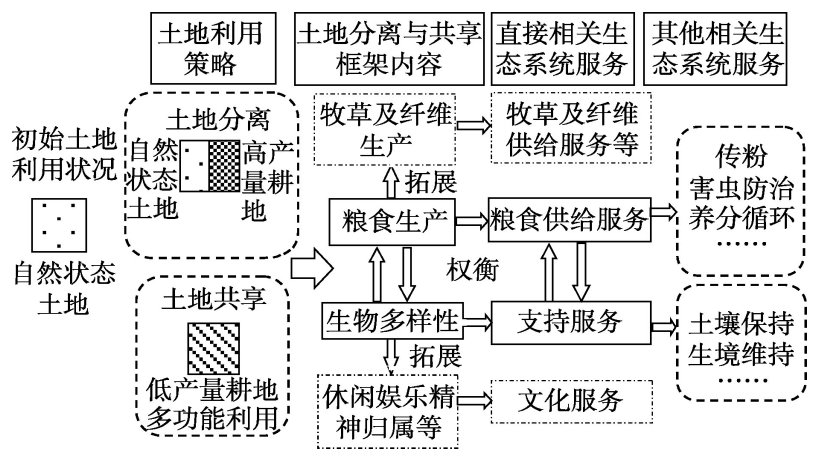

图 1 从生态系统服务视角解析 “土地分离与共享框架”

Fig.1 Land sparing versus sharing framework under a perspective from ecosystem services
服务视角出发, 可拓宽该框架中对生态系统状况的 评估手段。

\section{2 土地利用策略选择}

无论是土地分离还是土地共享策略,其初衷均 是在保证粮食生产的同时减少对生物多样性以及 生态系统服务的影响。从生物多样性的角度看, 尽 管有些种群的密度会随着粮食产量的增加而增大, 但总体而言,粮食供给服务的提升会对区域整体生 物多样性产生消极影响。

不同类型生态系统服务间关系较为复杂。若 某种生态系统服务和产品的增加,会导致其他服务 和产品的减少(Rodríguez et al, 2006; Bennett et al, 2007), 则称生态系统服务间存在权衡(trade-offs)关 系, 具体到本文中, 即粮食生产服务的增加, 导致生 物多样性或其他类型生态系统服务(以下简称“其 他服务”)的减少。图 2利用二维坐标系表示了这种 权衡关系。随着粮食生产服务的增加,有些服务呈 现加速度减少、凸向原点的衰减(图 2a); 有些服务呈 现加速度增加、凹向原点的衰减(图 2b)。根据构成 曲线的形状不同, 可分别命名为凸权衡(convex tradeoff) 和凹权衡 (concave tradeoff) (Lester et al, 2013)。其产生的原因与区域自然环境和社会经济 有关(Verburg et al, 2015), 如凸权衡多发生在生态 脆弱、恢复力差的地区, 凹权衡多发生在生态系统 较为稳健、恢复力强的地区(Aillery et al, 2001; Viglizzo et al, 2006; Phalan, Balmford et al, 2011)。凹权 衡状态下, 在粮食供给增加的初期对其他服务影响 不大, 当粮食供给增加到一定程度之后, 就会对其 他服务有很大影响; 凸权衡状态下, 在粮食供给增 加的初期即对生物多样性和其他服务有很大影 响。权衡关系的凹凸特性影响了土地利用策略的
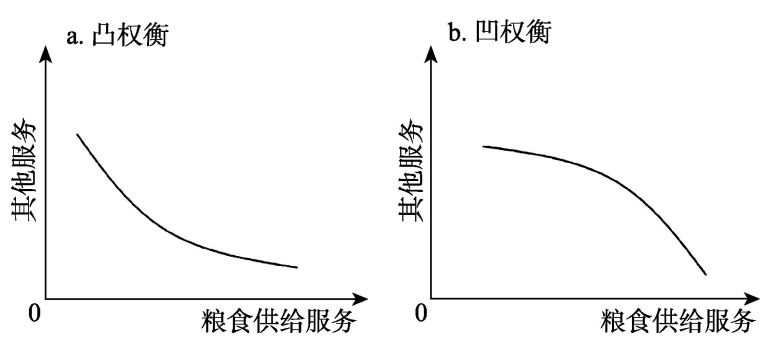

图 2 生物多样性/处于权衡关系的生态系统服务与 粮食供给服务间关系

Fig.2 Relationship between biodiversity/ecosystem services in trade-offs and food provision 
选择, 本文在如下基本假设下, 通过函数证明解释 策略的可能选择。

假设在原生土地状态下, 单位面积粮食供给服 务为 $D_{0}$; 在土地共享策略中, 低强度耕种时供给服 务强度为 $D_{\mathrm{L}}$; 高强度耕种时供给服务强度为 $D_{\mathrm{H}}$, 且 有 $D_{0}<D_{\mathrm{L}}<D_{\mathrm{H}}$; 单位面积其他服务是与粮食供给服 务强度相关的函数 $f(D)$; 同时假设人类对于其他服 务为线性的无偏好选择,即其他服务从 1 增加到 2 , 与 100 增加到 101 的收益相同。在初始状态、土地 共享策略中低强度耕种时、土地分离策略中高强 度耕种时, 其他服务量分别为 $f\left(D_{0}\right) 、 f\left(D_{\mathrm{L}}\right)$ 和 $f\left(D_{\mathrm{H}}\right)$, 且 $f\left(D_{0}\right)>f\left(D_{\mathrm{L}}\right)>f\left(D_{\mathrm{H}}\right)$ 。土地总量为 1 , 在土地分离策 略时, 保留原生态土地总量的面积为 $x$, 则进行高强 度耕种的土地面积为 1- $x$; 在实行土地共享策略时 土地总量也为 1 。

实行土地共享策略时, 其他服务总量为 $f\left(D_{\mathrm{L}}\right)$, 粮食供给服务总量为 $D_{\mathrm{L}}$; 在实行土地分离策略时, 其他服务总量为 $(1-x) f\left(D_{\mathrm{H}}\right)+x f\left(D_{0}\right)$, 粮食供给服务总 量为 $(1-x) D_{\mathrm{H}}+x D_{0}$; 因此, 在两种策略均能保证粮食 供给服务的总量不下降 $(1-x) D_{\mathrm{H}}+x D_{0}=D_{\mathrm{L}}$ 这一约束 下, 比较 $f\left(D_{\mathrm{L}}\right)$ 与 $(1-x) f\left(D_{\mathrm{H}}\right)+x f\left(D_{0}\right)$ 的大小即可判断 哪种策略更为适宜。

\subsection{1 生态脆弱区策略选择}

在生态脆弱区域, 函数形状为凸向原点, 即从 $D_{0}$ 到 $D_{\mathrm{L}}$ 点的减少速率大于从 $D_{\mathrm{L}}$ 到 $D_{\mathrm{H}}$ 点, 即有

$$
\frac{f\left(D_{\mathrm{H}}\right)-f\left(D_{\mathrm{L}}\right)}{D_{\mathrm{H}}-D_{\mathrm{L}}}>\frac{f\left(D_{\mathrm{L}}\right)-f\left(D_{0}\right)}{D_{\mathrm{L}}-D_{0}}
$$

式中: $D_{0}$ 为原生土地状态下单位面积粮食供给服 务; $D_{\mathrm{L}}$ 为土地共享策略中低强度耕种时供给服务强 度; $D_{\mathrm{H}}$ 为土地分离策略中高强度耕种时供给服务强 度; $f\left(D_{0}\right) 、 f\left(D_{\mathrm{L}}\right)$ 和 $f\left(D_{\mathrm{H}}\right)$ 分别为初始状态、土地共享 策略中低强度耕种时、土地分离策略中高强度耕种 时其他服务量(以下各公式同)。

由于 $D_{\mathrm{L}}-D_{0}<0$, 则:

$$
\frac{D_{\mathrm{L}}-D_{0}}{D_{\mathrm{H}}-D_{\mathrm{L}}}<\frac{f\left(D_{\mathrm{L}}\right)-f\left(D_{0}\right)}{f\left(D_{\mathrm{H}}\right)-f\left(D_{\mathrm{L}}\right)}
$$

由约束条件有:

$$
\frac{1-x}{x}=\frac{D_{\mathrm{L}}-D_{0}}{D_{\mathrm{H}}-D_{\mathrm{L}}}
$$

式中: $x$ 为土地分离策略时, 保留原生态土地总量的 面积(以下各公式同)。联立式(2)和式(3)为:

$$
\frac{1-x}{x}<\frac{f\left(D_{\mathrm{L}}\right)-f\left(D_{0}\right)}{f\left(D_{\mathrm{H}}\right)-f\left(D_{\mathrm{L}}\right)}
$$

由于 $f\left(D_{\mathrm{L}}\right)-f\left(D_{0}\right)<0$, 则有:

$$
(1-x)\left(f\left(D_{\mathrm{H}}\right)-f\left(D_{\mathrm{L}}\right)\right)>x\left(f\left(D_{\mathrm{L}}\right)-f\left(D_{0}\right)\right)
$$

进而, $(1-x) f\left(D_{\mathrm{H}}\right)+x f\left(D_{0}\right)>f\left(D_{\mathrm{L}}\right)$

即在生态系统较为脆弱的地区, 土地分离策略 较为适宜(图 3), 在粮食产量相同的情况下, 其他服 务更高, 应单辟出一块自然保护区用以保护生物多 样性。

\subsection{2 生态稳健区策略选择}

在生态稳健区域, 函数形状为凹向原点, 即从 $D_{0}$ 到 $D_{\mathrm{L}}$ 点的减少速率小于从 $D_{\mathrm{L}}$ 到 $D_{\mathrm{H}}$ 点, 即有:

$$
\frac{f\left(D_{\mathrm{H}}\right)-f\left(D_{\mathrm{L}}\right)}{D_{\mathrm{H}}-D_{\mathrm{L}}}<\frac{f\left(D_{\mathrm{L}}\right)-f\left(D_{0}\right)}{D_{\mathrm{L}}-D_{0}}
$$

由于 $D_{\mathrm{L}}-D_{0}<0$, 则:

$$
\frac{D_{\mathrm{L}}-D_{0}}{D_{\mathrm{H}}-D_{\mathrm{L}}}>\frac{f\left(D_{\mathrm{L}}\right)-f\left(D_{0}\right)}{f\left(D_{\mathrm{H}}\right)-f\left(D_{\mathrm{L}}\right)}
$$

联立约束条件式(3)和式(7)为:

$$
\frac{1-x}{x}>\frac{f\left(D_{\mathrm{L}}\right)-f\left(D_{0}\right)}{f\left(D_{\mathrm{H}}\right)-f\left(D_{\mathrm{L}}\right)}
$$

由于 $f\left(D_{\mathrm{L}}\right)-f\left(D_{0}\right)<0$, 则有:

$$
(1-x)\left(f\left(D_{\mathrm{H}}\right)-f\left(D_{\mathrm{L}}\right)\right)<x\left(f\left(D_{\mathrm{L}}\right)-f\left(D_{0}\right)\right)
$$

进而, $(1-x) f\left(D_{\mathrm{H}}\right)+x f\left(D_{0}\right)<f\left(D_{\mathrm{L}}\right)$

即在生态系统较为稳健的地区, 土地共享策略 较为适宜(图 4), 在粮食产量相同的情况下, 选择土 地共享策略则其他服务更高。

综上, 土地分离与共享策略的选取需要考虑生 态系统异质性, 取决于生物多样性或其他服务对以 粮食生产为代表的人类活动的响应。在判断区域 应采取哪一种策略时,应先判断当地的生态系统服 务间权衡关系属于凸权衡还是凹权衡。

\section{4 基于生态系统服务视角的“土地共 享”工程解析}

“土地分离与共享框架” 自 2005 年提出以来,已 应用于土地科学(von Wehrden et al, 2014)、保护生 物学(Benayas et al, 2012; Quinn et al, 2012; Macchi et al, 2013)以及生态学(Bommarco et al, 2013)等多 个领域。Barral等(2015)对全球 54 个“土地共享”或 “土地分离”生态恢复工程案例进行了分析。在中 国的土地利用实践中, “土地共享”策略应用较少, 因此文本以美国典型土地共享工程为例, 基于生态 系统服务视角分析工程实施的效果, 为扩大土地利 用策略选择空间,更有效利用土地提供参考。 


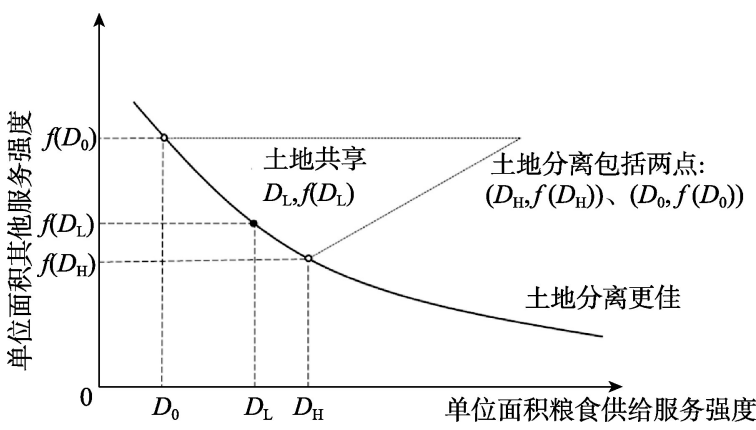

图 3 生态脆弱地区的策略选择

Fig.3 Choice of strategy in ecologically vulnerable areas

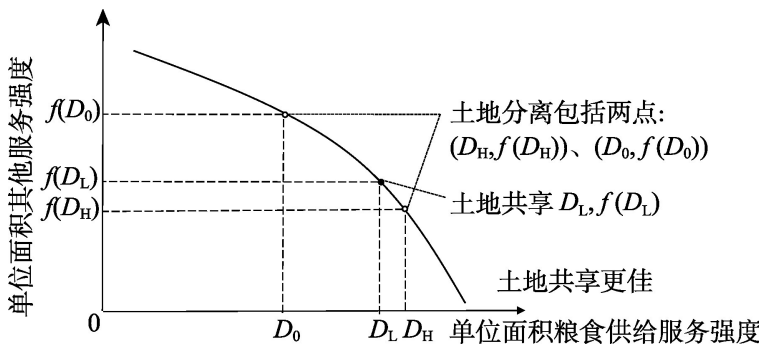

图 4 生态系统稳健地区的策略选择

Fig.4 Choice of strategy in ecologically stable areas
在 20 世纪 80 年代中期, 美国启动了 “保护储备 计划”(USDA Conservation Reserve Program, 以下简 称 “工程”)(United States Department of Agriculture Farm Service Agency, 2008)的土地共享策略,鼓励 农民采用天然植被与耕地交错的方式,将田块边 缘、水域边缘,或者是农田中部分易侵蚀的耕地转 变为林地、草地等天然植被, 以减少土壤侵蚀、增强 地下水补给、提高水质,增加野生动物栖息地。这 一计划的实施引发了学界的广泛关注, 取得了众多 研究成果, 为从生态系统服务视角分析“土地共享” 策略提供了素材。这一计划的分析成果,也将为中 国类似工程的实施提供参考。

通过文献搜集和整理(表 1),使用图 1 的框架对 该工程进行分析。从实施的目的上看,工程旨在保 护和提升当地的土壤保持、水源涵养等生态系统服 务, 并在一定程度上解决粮食产能过剩问题。工程 实施后,不少研究表明工程达到了预期效果。在生 物多样性方面, 工程实施对项目区内生物多样性产 生了积极影响; 但有研究发现(O'Connell et al, 2012), 实施土地共享策略后, 由于地表径流的截 留, 会造成项目区下游湿地水量减少, 生物多样性

表 1 基于生态系统服务视角的美国典型土地共享框架解析

Tab.1 Analysis of the Conservation Reserve Program in the United States from ecosystem service perspective

\begin{tabular}{|c|c|c|c|c|c|c|c|c|c|}
\hline 策略项目 & 概述 & \multicolumn{2}{|c|}{$\begin{array}{l}\text { 生物多样性或 } \\
\text { 生态系统服务 }\end{array}$} & 变化 & 采样地 & $\begin{array}{c}\text { 生态系统 } \\
\text { 状况 }\end{array}$ & $\begin{array}{c}\text { 与粮食供给服务 } \\
\text { 权衡/协同状态 }\end{array}$ & $\begin{array}{l}\text { 理想策略 } \\
\text { 选择 }\end{array}$ & 文献 \\
\hline \multirow{16}{*}{$\begin{array}{cc} & \text { 保 } \\
\text { 土 } & \text { 护 } \\
\text { 地 } & \text { 储 } \\
\text { 共 } & \text { 备 } \\
\text { 享 } & \text { 计 } \\
& \text { 划 }\end{array}$} & \multirow{16}{*}{$\begin{array}{l}1985 \text { 年 } \\
\text { 启动, 对 } \\
\text { 象为美 } \\
\text { 国范围 } \\
\text { 内自愿 } \\
\text { 参与的 } \\
\text { 农户 }\end{array}$} & \multicolumn{2}{|c|}{ 生物多样性 } & 增加 & 阿拉斯加腹地 & 脆弱 & 权衡 & 分离 & Seefeldt et al, 2010 \\
\hline & & & & 周边地区减少 & 新墨西哥等 6州 & 稳健 & 协同 & - & O'Connell et al, 2012 \\
\hline & & \multirow{4}{*}{$\begin{array}{l}\text { 供给 } \\
\text { 服务 }\end{array}$} & 粮食供给 & 减少 & 科罗拉多州柯林斯堡 & 稳健 & - & 共享 & Reeder et al, 1998 \\
\hline & & & & & 北达科塔州 16 县 & 稳健 & - & 共享 & Bangsund et al, 2004 \\
\hline & & & $\begin{array}{l}\text { 生物能源 } \\
\text { 材料供给 }\end{array}$ & 增加 & 堪萨斯州瓦利福尔斯 & 稳健 & 权衡 & 共享 & Xu et al, 2014 \\
\hline & & & 水供给 & 增加 & $\begin{array}{l}\text { 俄克拉荷马州得克萨 } \\
\text { 斯县 }\end{array}$ & 稳健 & 权衡 & 共享 & Rao et al, 2010 \\
\hline & & \multirow{6}{*}{$\begin{array}{l}\text { 调节 } \\
\text { 服务 }\end{array}$} & 水质净化 & 增加 & 阿拉斯加腹地 & 脆弱 & 权衡 & 共享 & Ribaudo, 1989 \\
\hline & & & & 增加 & 伊利诺伊州费耶特县 & 稳健 & 权衡 & 共享 & Lant, 1991 \\
\hline & & & 土壤保持 & 增加 & 阿拉斯加腹地 & 脆弱 & 权衡 & 共享 & Seefeldt et al, 2010 \\
\hline & & & & 增加 & 堪萨斯州西南部六县 & 稳健 & 权衡 & 共享 & Park et al, 2005 \\
\hline & & & 野生动物 & 增加 & 阿拉斯加腹地 & 脆弱 & 权衡 & 共享 & Seefeldt et al, 2010 \\
\hline & & & 栖息地 & 增加 & 堪萨斯州芬尼县 & 稳健 & 权衡 & 共享 & Egbert et al, 2002 \\
\hline & & \multirow{3}{*}{$\begin{array}{l}\text { 支持 } \\
\text { 服务 }\end{array}$} & 土壤碳 & 增加 & 科罗拉多州柯林斯堡 & 稳健 & 权衡 & 共享 & Reeder et al, 1998 \\
\hline & & & 土壤磷 & 增加 & $\begin{array}{l}\text { 内布拉斯加州盖奇县 } \\
\text { 和萨利内县 }\end{array}$ & 稳健 & 权衡 & 共享 & Bach et al, 2010 \\
\hline & & & 土壤氮 & 增加 & 科罗拉多州柯林斯堡 & 稳健 & 权衡 & 共享 & Reeder et al, 1998 \\
\hline & & $\begin{array}{l}\text { 文化 } \\
\text { 服务 }\end{array}$ & 娱乐狩猎 & 增加 & 北达科塔州 16 县 & 稳健 & 权衡 & 共享 & Bangsund et al, 2004 \\
\hline
\end{tabular}


减少。在粮食供给方面, 美国实施土地共享策略 后, 粮食产量呈现下降趋势(Egbert et al, 2002; Rao et al, 2010)。在原材料供给方面,美国依靠技术优 势, 从地表覆被增加的植物生物量中获取了大量的 纤维素生物质能源(Xu et al, 2014)。土壤保持、水 源涵养等调节服务以及土壤碳、氮、磷等支持服务 总量也有所增加。如土壤养分方面, 1985-2004年 美国内布拉斯加州的盖奇县和萨利内县的土壤大 颗粒物形成增加了 $33 \%$, 土壤氮磷总量也有明显提 升(Reeder et al, 1998), 同时由于野生动物数量增 多, 人们有了更多娱乐性的狩猎机会 (Egbert et al, 2002), 文化服务也有所加强。

从策略选取上看, 工程实施前美国面临粮食产 量过剩问题, 在文献中获取的项目实施地多属于生 态稳健的区域, 粮食供给服务与生物多样性/其他处 于权衡状态的生态系统服务间处于凹权衡状态, 按 照图 4 所示的分析, 采用土地共享策略较为适宜。 美国的“保护储备计划”土地共享策略在实践中也 确实产生了较好的效果。

通过对美国典型土地共享工程的分析可以看 出, 从生态系统服务的视角出发解析土地分离与共 享策略, 有助于全面审视工程实施成果, 特别是工 程外部性效应。由于生态系统的复杂性、流动性和 尺度依存的特性, 局部实施的工程可能对周边地区 或更大范围内的生态环境产生影响,如美国“保护 储备计划”对项目区下游湿地生物多样性的负面影 响。土地较为贫痊和生态脆弱的地区生产能力有 限, 土地分离策略增加了高产量耕地面积, 盲目增 加这类区域的耕地生产率可能会造成土壤有机质 下降, 但土地肥沃、生产力高的地区则不存在这类 问题(Ramankutty et al, 2012)。在中国土地利用规 划与调整的过程中, 也应充分考虑生态系统的脆弱 性, 在土地利用策略选择时参考国际经验, 采取适 宜的策略, 如划分禁耕区或在生态脆弱区域建立单 独的自然保护区等。

\section{5 结论与讨论}

\section{1 结论}

本文介绍了 “土地分离与共享框架” 的内涵和 研究进展; 分析了这一框架中所蕴含的生态系统服 务权衡关系, 并从权衡关系的表现形式出发, 归纳 了不同土地利用策略的应用条件; 结合美国典型土
地共享案例, 阐释了土地共享策略对粮食生产等生 态系统服务的影响。其结论主要包括:

(1) 人类对土地的需求从单一的粮食供给服务 需求演进到粮食供给服务和其他生态系统服务的 综合需求,促使 “土地分离与共享”框架在生态系统 服务的视角下进行拓展和深人挖掘。

(2) 当粮食供给服务与其他服务处于权衡状态 时,在生态脆弱、恢复力差的地区, 不同生态系统服 务多表现为凸权衡关系,一般适宜采用分离策略; 在生态系统稳定、恢复力强的地区, 不同生态系统 服务多表现为凹权衡关系,一般适宜采用共享策 略。从生态系统服务的视角看, 美国土地利用实践 选择土地共享策略是比较适宜的。

(3) 土地利用是多要素综合影响的结果, 在需 要全面考虑策略实施地的实际情况。生态系统服 务可在策略选择和评估实施效果中发挥评估工具 的作用,为促进区域生态文明建设和可持续发展提 供帮助。

\section{2 讨论}

(1) 解析框架的难点。从生态系统服务的视角 拓展 “土地分离与共享框架”, 并将其运用在生态工 程的效用评估中, 有助于从宏观层面把握重大生态 工程的顶层设计。判断两种或多种生态系统服务 之间处于凸权衡或凹权衡关系是策略选择的关键， 也是生态系统服务领域的热点和难点问题。现阶 段由于生态系统服务研究本身有待完善,生态系统 服务和土地利用之间, 以及不同生态系统服务之间 的关系尚未完全厘清, 人类对于生态系统服务的需 求和评价体系也没有形成共识, 因此制定以多种生 态系统服务共赢为目标的土地利用策略还有较大 的研究空间。

(2) 框架自身局限。在“土地分离与共享框架” 的早期研究中, “分离”或“共享”被视为非黑即白的 二分选择,但越来越多的研究发现, 面对高度复杂 的生态系统, 这并非“是”或“非”的互斥策略(Ramankutty et al, 2013), 其中存在着灰色地带, 可以相 互融合(Lusiana et al, 2012)。例如在“分离”策略中, 可在保护区或禁耕区内继续划分出完全禁止区和 可“共享”使用区域,这与自然保护区中“核心区”和 “实验区”的划分较为相似。土地“分离”强调从时 间上给予生态环境充分的恢复，即时间上的集约， 而“共享”侧重于释放更多的空间减少人类干扰来 保护生态环境,因此在土地的集约利用中,也应同 
时考虑分离与共享的策略(Minang et al, 2013; 朱会 义等, 2014)。此外, 也有学者提出在农业耕作的最 初即优化土地配置, 比随后实施“分离”或“共享”策 略更有助于区域的可持续发展(Law et al, 2015)。

(3) 对中国的启示。美国“土地保护储备计划” 的成功经验为其他国家提供了重要参考。由于各 国土地基础条件、权属关系和政策目标不尽相同, 因此在选择“土地分离”或是“土地共享”策略时,需 要有针对性的实践操作。西方国家多在私有土地 中推广使用土地共享策略, 因为政府无权限制私有 土地中哪些地区绝对禁止耕种。在中国现行土地 所有制度下, 政府推行土地分离策略执行效率更 高, 监督成本更小, 因此, 中国多实施以土地分离为 主的土地限制性开发政策来处理耕地和建设用地 间的博亦。在耕地和生态用地间的权衡中可采用 “土地共享”的策略, 发掘土地的复合功能, 使耕地 作为绿色空间提高生态系统服务的供给。

\section{参考文献(References)}

戴漂漂, 张旭珠, 刘云慧. 2015. 传粉动物多样性的保护与农 业景观传粉服务的提升[J]. 生物多样性, 23(3): 408-418.

[Dai P P, Zhang X Z, Liu Y H. 2015. Conserving pollinator diversity and improving pollination services in agricultural landscapes[J]. Biodiversity Science, 23(3): 408-418.]

傅伯杰. 2013. 生态系统服务与生态安全[M]. 北京: 高等教

育出版社. [Fu B J. 2013. Ecosystem service and ecological security[M]. Beijing, China: Higher Education Press.]

傅伯杰, 张立伟. 2014. 土地利用变化与生态系统服务: 概 念、方法与进展 [J]. 地理科学进展, 33(4): 441-446. [Fu B J, Zhang L W. 2014. Land-use change and ecosystem services: Concepts, methods and progress[J]. Progress in Geography, 33(4): 441-446.]

刘卫. 2015. 广州古城水系与城市发展关系研究[D]. 广州: 华 南理工大学. [Liu W. 2015. Study on the relationship between urban canal-system of ancient Guangzhou and city development[D]. Guangzhou, China: South China University of Technology.]

朱会义, 孙明慧. 2014. 土地利用集约化研究的回顾与未来 工作重点[J]. 地理学报, 69(9): 1346-1357. [Zhu H Y, Sun M H. 2014. Main progress in the research on land use intensification[J]. Acta Geographica Sinica, 69(9): 13461357.]

左太安. 2014. 西南喀斯特山区石漠化贫困效应研究: 以毕 节试验区为例 [D]. 重庆: 西南大学. [Zuo T A. 2014. Rocky desertification poverty effect in karst mountainous areas of Southwest China: A case study of Bijie experimental area[D]. Chongqing, China: Southwest University]
Aillery M, Shoemaker R, Caswell M. 2001. Agriculture and ecosystem restoration in South Florida: Assessing tradeoffs from water-retention development in the Everglades agricultural area[J]. American Journal of Agricultural Economics, 83(1): 183-195.

Bach E M, Baer S G, Meyer C K, et al. 2010. Soil texture affects soil microbial and structural recovery during grassland restoration[J]. Soil Biology and Biochemistry, 42(12): 2182-2191.

Bangsund D A, Hodur N M, Larry L F. 2004. Agricultural and recreational impacts of the conservation reserve program in rural North Dakota, USA[J]. Journal of Environmental Management, 71(4): 293-303.

Barral M P, Benayas J M R, Meli P, et al. 2015. Quantifying the impacts of ecological restoration on biodiversity and ecosystem services in agroecosystems: A global meta-analysis[J]. Agriculture, Ecosystems \& Environment, 202: 223 231.

Benayas J M R, Bullock J M. 2012. Restoration of biodiversity and ecosystem services on agricultural land[J]. Ecosystems, 15(6): 883-899.

Bennett E M, Balvanera P. 2007. The future of production systems in a globalized world[J]. Frontiers in Ecology and the Environment, 5(4): 191-198.

Boisvert V. 2015. Conservation banking mechanisms and the economization of nature: An institutional analysis[J]. Ecosystem Services, 15: 134-142.

Bommarco R, Kleijn D, Potts S G. 2013. Ecological intensification: Harnessing ecosystem services for food security[J]. Trends in Ecology and Evolution, 28(4): 230-238.

Cardador L, de Cáceres M, Giralt D, et al. 2015. Tools for exploring habitat suitability for steppe birds under land use change scenarios[J]. Agriculture, Ecosystems \& Environment, 200: 119-125.

Costanza R, d'Arge R, de Groot R, et al. 1997. The value of the world's ecosystem services and natural capital[J]. Nature, 387: 253-260.

Cunningham S A, Attwood S J, Bawa K S, et al. 2013. To close the yield-gap while saving biodiversity will require multiple locally relevant strategies[J]. Agriculture, Ecosystems \& Environment, 173: 20-27.

Edwards D P, Hodgson J A, Hamer K C, et al. 2010. Wildlifefriendly oil palm plantations fail to protect biodiversity effectively[J]. Conservation Letters, 3(4): 236-242.

Egan J F, Mortensen D A. 2012. A comparison of land-sharing and land-sparing strategies for plant richness conservation in agricultural landscapes[J]. Ecological Applications, 22 (2): 459-471.

Egbert S L, Park S, Price K P, et al. 2002. Using conservation 
reserve program maps derived from satellite imagery to characterize landscape structure[J]. Computers and Electronics in Agriculture, 37(1-3): 141-156.

Fischer J, Abson D J, Butsic V, et al. 2014. Land sparing versus land sharing: Moving forward $[\mathrm{J}]$. Conservation Letters, 7(3): 149-157.

Fischer J, Brosi B, Daily G C, et al. 2008. Should agricultural policies encourage land sparing or wildlife-friendly farming $[\mathrm{J}]$. Frontiers in Ecology and the Environment, 6(7): 380-385.

Green R E, Cornell S J, Scharlemann J P W, et al. 2005. Farming and the fate of wild nature[J]. Science, 307: 550-555.

Habel J C, Weisser W W, Eggermont H, et al. 2013. Food security versus biodiversity protection: An example of landsharing from East Africa[J]. Biodiversity and Conservation, 22(6-7): 1553-1555.

Huang J, Tichit M, Poulot M, et al. 2015. Comparative review of multifunctionality and ecosystem services in sustainable agriculture $[\mathrm{J}]$. Journal of Environmental Management, 149: 138-147.

Lant C L. 1991. Potential of the Conservation Reserve Program to control agricultural surface water pollution[J]. Environmental Management, 15(4): 507-518.

Law E A, Meijaard E, Bryan B A, et al. 2015. Better land-use allocation outperforms land sparing and land sharing approaches to conservation in Central Kalimantan, Indonesia [J]. Biological Conservation, 186: 276-286.

Lerouge F, Sannen K, Gulinck H, et al. 2016. Revisiting production and ecosystem services on the farm scale for evaluating land use alternatives[J]. Environmental Science \& Policy, 57: 50-59.

Lester S E, Costello C, Halpern B S, et al. 2013. Evaluating tradeoffs among ecosystem services to inform marine spatial planning[J]. Marine Policy, 38: 80-89.

Lusiana B, van Noordwijk M, Cadisch G. 2012. Land sparing or sharing: Exploring livestock fodder options in combination with land use zoning and consequences for livelihoods and net carbon stocks using the FALLOW model[J]. Agriculture, Ecosystems \& Environment, 159: 145-160.

Macchi L, Grau H R, Zelaya P V, et al. 2013. Trade-offs between land use intensity and avian biodiversity in the Dry Chaco of Argentina: A tale of two gradients[J]. Agriculture, Ecosystems \& Environment, 174: 11-20.

Merckx T, Pereira H M. 2014. Reshaping agri-environmental subsidies: From marginal farming to large-scale rewilding [J]. Basic and Applied Ecology, 16(2): 95-103.

Millennium Ecosystem Assessment. 2005. Ecosystems and human well-being: Biodiversity synthesis report[R]. Washington DC: Island Press.
Minang P A, van Noordwijk M. 2013. Design challenges for achieving reduced emissions from deforestation and forest degradation through conservation: Leveraging multiple paradigms at the tropical forest margins[J]. Land Use Policy, 31: 61-70.

Montoya-Molina S, Giraldo-Echeverri C, Montoya-Lerma J, et al. 2016. Land sharing vs. land sparing in the dry Caribbean lowlands: A dung beetles' perspective[J]. Applied Soil Ecology, 98: 204-212.

Newbold T, Hudson L N, Phillips H R P, et al. 2014. A global model of the response of tropical and sub-tropical forest biodiversity to anthropogenic pressures $[\mathrm{J}]$. Proceedings of the Royal Society B: Biological Sciences, 281(1792): 20141371

O'Connell J L, Johnson L A, Smith L M, et al. 2012. Influence of land- use and conservation programs on wetland plant communities of the semiarid United States Great Plains[J]. Biological Conservation, 146(1): 108-115.

Park S, Egbert S L. 2005. Assessment of soil erodibility indices for conservation reserve program lands in southwestern Kansas using satellite imagery and GIS techniques[J]. Environmental Management, 36(6): 886-898.

Phalan B, Balmford A, Green R E, et al. 2011. Minimising the harm to biodiversity of producing more food globally $[\mathrm{J}]$ Food Policy, 36(S1): S62-S71.

Phalan B, Onial M, Balmford A, et al. 2011. Reconciling food production and biodiversity conservation: Land sharing and land sparing compared[J]. Science, 333: 1289-1291.

Quinn J E, Brandle J R, Johnson R J. 2012. The effects of land sparing and wildlife- friendly practices on grassland bird abundance within organic farmlands[J]. Agriculture, Ecosystems \& Environment, 161: 10-16.

Ramankutty N, Rhemtulla J. 2012. Can intensive farming save nature $[\mathrm{J}]$. Frontiers in Ecology and the Environment, 10 (9): 455

Ramankutty N, Rhemtulla J. 2013. Land sparing or land sharing: Context dependent[J]. Frontiers in Ecology and the Environment, 11(4): 178.

Rao M N, Yang Z M. 2010. Groundwater impacts due to conservation reserve program in Texas County, Oklahoma[J]. Applied Geography, 30(3): 317-328.

Reeder J D, Schuman G E, Bowman R A. 1998. Soil C and N changes on conservation reserve program lands in the Central Great Plains[J]. Soil and Tillage Research, 47(3-4): 339-349.

Ribaudo M O. 1989. Targeting the conservation reserve program to maximize water quality benefits[J]. Land Economics, 65(4): 320-332.

Rodríguez J P, Beard Jr T D, Bennett E M, et al. 2006. Trade- 
offs across space, time, and ecosystem services[J]. Ecology and Society, 11(1): 28.

Scariot A. 2013. Land sparing or land sharing: The missing link[J]. Frontiers in Ecology and the Environment, 11(4): 177-178.

Seefeldt S S, Conn J S, Zhang M C, et al. 2010. Vegetation changes in conservation reserve program lands in interior Alaska[J]. Agriculture, Ecosystems \& Environment, 135(12): 119-126.

United States Department of Agriculture Farm Service Agency (USDA FSA). 2008. Conservation Reserve Program: Summary and enrollment statistics[R]. Washington DC: USDA FSA: 7-9.

Verburg P H, Crossman N, Ellis E C, et al. 2015. Land system science and sustainable development of the earth system: A global land project perspective[J]. Anthropocene, 12: 29-
41.

Viglizzo E F, Frank F C. 2006. Land-use options for Del Plata Basin in South America: Tradeoffs analysis based on ecosystem service provision[J]. Ecological Economics, 57(1): 140-151.

von Wehrden H, Abson D J, Beckmann M, et al. 2014. Realigning the land-sharing/land-sparing debate to match conservation needs: Considering diversity scales and land-use history[J]. Landscape Ecology, 29(6): 941-948.

Xu F, Linnebur K, Wang D H. 2014. Torrefaction of conservation reserve program biomass: A techno-economic evaluation[J]. Industrial Crops and Products, 61: 382-387.

Yoshii C, Yamaura Y, Soga M, et al. 2015. Comparable benefits of land sparing and sharing indicated by bird responses to stand-level plantation intensity in Hokkaido, northern Japan[J]. Journal of Forest Research, 20(1): 167-174.

\title{
Land sparing versus sharing framework from ecosystem service perspective
}

\author{
FENG Zhe ${ }^{1,2}$, XU Xuegong ${ }^{2 *}$, ZHOU Jian ${ }^{3,4}$, GAO Yang ${ }^{3,4}$ \\ (1. School of Land Science and Technology, China University of Geosciences, Beijing 100083, China; \\ 2. College of Urban and Environmental Sciences, Peking University; Key Laboratory for Earth Surface Processes, \\ Ministry of Education, Beijing 100087, China; 3. Department of Land Resources Management, China Agricultural \\ University, Beijing 100193, China; 4. Key Laboratory of Agricultural Land Quality, Monitoring and Control,
}

Ministry of Land and Resources, Beijing 100193, China)

\begin{abstract}
Land sparing versus sharing framework is an important theory for balancing food production and biodiversity conservation for sustainable land use. In this article, the relationship between the "land sparing versus sharing framework" and ecosystem services was summarized by revealing the theoretical basis of the combination of ecosystem services and land sparing versus sharing framework. The impacts of land- use decisions such as the USDA Conservation Reserve Program on food production, biodiversity, and other direct and indirect ecosystem services were analyzed. The application of this framework in Chinese environmental conservation projects was discussed, aiming at providing a reference for the coupling of land-use optimization and ecosystem services provision. The results show that: (1) The framework of "land sparing versus sharing" was based on multiple demands of ecosystem services from cultivated land. From an ecosystem service perspective, expansion of the framework can be seen as demand change from food production alone to multiple demands containing food production and other ecosystem services. (2) When food production service and other services are in a state of trade- off, in ecologically fragile and low resilience areas, where the relationship between ecosystem services shows a "convex tradeoff," land sparing strategy is often appropriate; whereas land sharing strategy is appropriate in ecologically stable and high resilience areas where the relationship between ecosystem services shows a "concave tradeoff." (3) Land sharing optimizes the demand of ecosystem services, according to the study of the land-use practices in the United States. Assessment of ecosystem services can be used as a tool to measure the effect of land-use policies and strategies.
\end{abstract}

Key words: land sparing versus sharing; ecosystem services; biodiversity; food production; trade-offs 